\title{
Fermion's Tunnelling with Effects of Quantum Gravity
}

\author{
Deyou Chen, ${ }^{1}$ Houwen $\mathrm{Wu}^{2}{ }^{2}$ and Haitang Yang ${ }^{2}$ \\ ${ }^{1}$ Institute of Theoretical Physics, China West Normal University, Nanchong 637009, China \\ ${ }^{2}$ Center for Theoretical Physics, College of Physical Science and Technology, Sichuan University, Chengdu 610064, China
}

Correspondence should be addressed to Deyou Chen; dyouchen@gmail.com

Received 31 August 2013; Accepted 9 October 2013

Academic Editor: Chao-Qiang Geng

Copyright (C) 2013 Deyou Chen et al. This is an open access article distributed under the Creative Commons Attribution License, which permits unrestricted use, distribution, and reproduction in any medium, provided the original work is properly cited.

\begin{abstract}
In this paper, using Hamilton-Jacobi method, we address the tunnelling of fermions in a 4-dimensional Schwarzschild spacetime. Based on the generalized uncertainty principle, we introduce the influence of quantum gravity. After solving the equation of motion of the spin-1/2 field, we derive the corrected Hawking temperature. It turns out that the correction depends not only on the black hole's mass but also on the mass (energy) of emitted fermions. It is of interest that, in our calculation, the quantum gravity correction decelerates the temperature increase during the radiation explicitly. This observation then naturally leads to the remnants in black hole evaporation. Our calculation shows that the residue mass is $\gtrsim M_{p} / \beta_{0}$, where $M_{p}$ is the Planck mass and $\beta_{0}$ is a dimensionless parameter accounting for quantum gravity effects. The evaporation singularity is then avoided.
\end{abstract}

\section{Introduction}

Hawking radiation is described as a quantum tunnelling effects of particles at horizons of black holes [1-9]. With the consideration of the background variation in black hole evaporation, Parikh and Wilczek studied the tunnelling behaviors of massless scalar particles [3]. They derived the modified emission spectra for spherically symmetric black holes. The leading corrections to the Hawking temperature are found to be also dependent on the energy of emitted particles. This work was extended to massive and charged scalar particles. The Hawking radiation of general black holes was studied in $[4,5]$. For an outgoing massive particle, the equation of motion is different from that of a massless particle. The trajectory of massless particles is a null geodesic, while the massive particle's motion satisfies de Broglie wave and is the phase velocity of outgoing particles. Subsequently, the tunnelling behaviors of fermions were carefully investigated with Hamilton-Jacobi method by Kerner and Mann $[10,11]$. The Hawking temperatures were recovered by the fermions tunnelling. The extension of this work to complicated spacetimes is referred to in [12-15]. The noncommutative spacetimes bring a similar consequences in the spirit of black holes thermodynamics. In [16], the tunnelling process in the noncommutative Schwarzschild black holes was researched by Parikh-Wilczek tunnelling method. They derived an interesting result that information might be preserved by a stable black hole remnant.

Various theories of quantum gravity predict the existence of a minimum measurable length [17-21]. This length can be approached from the generalized uncertainty principle (GUP). Through the modified fundamental commutation relation [22]

$$
\left[x_{i}, p_{j}\right]=i \hbar \delta_{i j}\left[1+\beta p^{2}\right] \text {, }
$$

the expression of GUP is derived as $\Delta x \Delta p \geq(\hbar / 2)[1+$ $\beta(\Delta p)^{2}$ ], where $\beta=\beta_{0} / M_{p}^{2} . M_{p}$ is the Planck mass. $\beta_{0}$ is a dimensionless parameter. From simple electroweak consideration, it is readily to find an upper limit $\beta_{0}<10^{34} . x_{i}$ and $p_{i}$ are defined by $x_{i}=x_{0 i}$ and $p_{i}=p_{0 i}\left(1+\beta p^{2}\right)$, respectively. $x_{0 i}$ and $p_{0 j}$ satisfy the canonical commutation relations $\left[x_{0 i}, p_{0 j}\right]=i \hbar \delta_{i j}$. The modification of the fundamental commutation relation is not unique. Other modifications are referred to in [23-25] and references therein.

These modifications are widely applied to gain some information about the quantum properties of gravity. Black holes are effective modes to explore the effects of quantum gravity. Incorporating effects of quantum gravity into black hole physics by GUP, some interesting implications and results were achieved [26-32]. It is shown in [26] that a small black hole is unstable. Moreover, the constraint for 
a large black hole comparable to the size of the cavity in connection with the critical mass is needed. In [27], the tunnelling radiation in the noncommutative higher spacetime was discussed with GUP. They found that information may be preserved in a stable black hole remnant. The characteristic size in the absorption process, represented by the black hole irreducible mass, was gotten in [28]. The remnant mass and corrections to the area law and heat capacity were obtained in [29]. In [30], following Parikh-Wilczek tunnelling method, based on GUP, the radiation of massless scalar particles in the Schwarzschild black hole was discussed. The commutation relation between the radial coordinate and the conjugate momentum are modified with GUP. The authors treat the natural cutoffs as a minimal length, a minimal momentum, and a maximal momentum. They addressed the tunnelling rate of black holes. The corrected Hawking temperature was obtained and related to the energy of emitted particles.

The purpose of this paper is to investigate fermions' tunnelling behavior cross the event horizon of a 4-dimensional Schwarzschild black hole, where the effects of quantum gravity are taken into account. We first modify the Dirac equation in curved spacetime to reflect the influence of quantum gravity. The model we adopt is the generalized uncertainty principle. We use the Hamilton-Jacob method to solve the equation of motion of the spinor field. Then, the tunnelling rate and Hawking temperature are calculated. Our results show that the quantum correction to the Hawking temperature is dependent not only on the black hole's mass but also on the mass and energy of emitted fermions. Moreover, the correction slows down the temperature increase during the evaporation. This in turn leads to the remnants in black hole evaporation and prevents the existence of the thermodynamics singularity.

The rest of the paper is organized as follows. In Section 2, taking into account the effects of quantum gravity, we modify the Dirac equation in curved spacetime by GUP and get a generalized Dirac equation. In Section 3, the fermion tunnelling behavior in the Schwarzschild black hole is addressed and the corrected Hawking temperature is derived. Section 4 is devoted to our discussion and conclusion.

\section{Generalized Dirac Equation in Curved Spacetime}

To take into account the effects of quantum gravity, we adopt the generalized commutation relation in [22] to modify the Dirac equation. In (1), the momentum operators are defined by

$$
p_{i}=p_{0 i}\left(1+\beta p^{2}\right) .
$$

The square of momentum operators is

$$
\begin{aligned}
p^{2} & =p_{i} p^{i}=-\hbar^{2}\left[1-\beta \hbar^{2}\left(\partial_{j} \partial^{j}\right)\right] \partial_{i} \cdot\left[1-\beta \hbar^{2}\left(\partial^{j} \partial_{j}\right)\right] \partial^{i} \\
& \simeq-\hbar^{2}\left[\partial_{i} \partial^{i}-2 \beta \hbar^{2}\left(\partial^{j} \partial_{j}\right)\left(\partial^{i} \partial_{i}\right)\right],
\end{aligned}
$$

where in the last step, we only keep the leading order term of $\beta$. To account for the effects from quantum gravity, the frequency is generalized as [33]

$$
\bar{\omega}=E\left(1-\beta E^{2}\right),
$$

with the energy operator $E=i \hbar \partial_{0}$. Substituting the mass shell condition $p^{2}+m^{2}=E^{2}$, we get the generalized expression of energy $[34,35]$ :

$$
\bar{E}=E\left[1-\beta\left(p^{2}+m^{2}\right)\right] .
$$

The tunnelling of massless scalar particles in the Schwarzschild black hole was studied in detail and the corrected Hawking temperature was derived in [30]. On the other hand, the Dirac equation with the consequence of GUP in flat spacetime has been investigated in [34].

We start with the Dirac equation in curved spacetime,

$$
i \gamma^{\mu}\left(\partial_{\mu}+\Omega_{\mu}\right) \psi+\frac{m}{\hbar} \psi=0, \quad \Omega_{\mu} \equiv \frac{i}{2} \omega_{\mu}^{a b} \Sigma_{a b}
$$

where $\omega_{\mu}{ }^{a b}$ is the spin connection defined by the tetrad $e^{\lambda}{ }_{b}$ and ordinary connection

$$
\omega_{\mu b}^{a}=e_{\nu}^{a} e_{b}^{\lambda} \Gamma_{\mu \lambda}^{\nu}-e_{b}^{\lambda} \partial_{\mu} e_{\lambda}^{a} .
$$

The Latin indices live in the flat metric $\eta_{a b}$ while Greek indices are raised and lowered by the curved metric $g_{\mu \nu}$. The tetrad can be constructed from

$$
\begin{aligned}
g_{\mu \nu} & =e_{\mu}{ }^{a} e_{\nu}{ }^{b} \eta_{a b}, \quad \eta_{a b}=g_{\mu \nu} e_{a}^{\mu} e_{b}{ }_{b}, \\
e^{\mu}{ }_{a} e_{\nu}{ }^{a} & =\delta_{\nu}^{\mu}, \quad e^{\mu}{ }_{a} e_{\mu}{ }^{b}=\delta_{a}^{b} .
\end{aligned}
$$

Back in (6), $\Sigma_{a b}$ 's are the Lorentz spinor generators defined by

$$
\Sigma_{a b}=\frac{i}{4}\left[\gamma^{a}, \gamma^{b}\right], \quad\left\{\gamma^{a}, \gamma^{b}\right\}=2 \eta^{a b}
$$

Then, one can construct the $\gamma^{\mu}$ 's in curved spacetime as

$$
\gamma^{\mu}=e_{a}^{\mu} \gamma^{a}, \quad\left\{\gamma^{\mu}, \gamma^{\nu}\right\}=2 g^{\mu \nu} .
$$

To get the generalized Dirac equation in curved spacetime, we rewrite (6) as

$$
-i \gamma^{0} \partial_{0} \psi=\left(i \gamma^{i} \partial_{i}+i \gamma^{\mu} \Omega_{\mu}+\frac{m}{\hbar}\right) \psi
$$

where $i=1,2, \ldots$ denotes the spatial coordinates. The lefthand side of the equation above is related to the energy. Using the generalized expression of energy (5) and the square of momentum operators (3), only keeping the leading order term of $\beta$, we get

$$
-i \gamma^{0} \partial_{0} \psi=\left(i \gamma^{i} \partial_{i}+i \gamma^{\mu} \Omega_{\mu}+\frac{m}{\hbar}\right)\left(1+\beta \hbar^{2} \partial_{j} \partial^{j}-\beta m^{2}\right) \psi .
$$


Therefore, the generalized Dirac equation in curved spacetime can be written as

$$
\begin{aligned}
& {\left[i \gamma^{0} \partial_{0}+i \gamma^{i} \partial_{i}\left(1-\beta m^{2}\right)+i \gamma^{i} \beta \hbar^{2}\left(\partial_{j} \partial^{j}\right) \partial_{i}\right.} \\
& +\frac{m}{\hbar}\left(1+\beta \hbar^{2} \partial_{j} \partial^{j}-\beta m^{2}\right) \\
& \left.+i \gamma^{\mu} \Omega_{\mu}\left(1+\beta \hbar^{2} \partial_{j} \partial^{j}-\beta m^{2}\right)\right] \psi=0 .
\end{aligned}
$$

This is the equation we are going to solve in the next section.

\section{Fermion's Tunnelling with Effects of Quantum Gravity}

In this section, we address the tunnelling behavior of spin- $1 / 2$ fermions across the event horizon of the Schwarzschild black hole. Effects of quantum gravity are taken into account. The metric is given by

$$
d s^{2}=-f(r) d t^{2}+\frac{1}{g(r)} d r^{2}+r^{2}\left(d \theta^{2}+\sin ^{2} \theta d \phi^{2}\right)
$$

with $f(r)=g(r)=1-(2 M / r)$, and $M$ is the black hole's mass. We have set $G=c=1$. The event horizon is located at $r_{h}=2 M$. The fermion motion is determined by the generalized Dirac equation (13). For a spin-1/2 particle, there are two states corresponding, respectively, to spin up and spin down. Following the standard ansatz, to describe the motion semiclassically, we assume the wave function of the spin up state as

$$
\Psi=\left(\begin{array}{c}
A \\
0 \\
B \\
0
\end{array}\right) \exp \left(\frac{i}{\hbar} I(t, r, \theta, \phi)\right),
$$

where $A, B$, and $I$ are functions of coordinates $t, r, \theta$, and $\phi$ and $I$ is the action of the emitted fermions. The process of spin down is the same as that of spin up. To solve (13), one should choose appropriate gamma matrices by exploiting (8)-(10). It is straightforward to guess a tetrad for the metric (14):

$$
e_{\mu}{ }^{a}=\operatorname{diag}\left(\sqrt{f}, \frac{1}{\sqrt{g}}, r, r \sin \theta\right) \text {. }
$$

Then, our gamma matrices are given by

$$
\begin{array}{ll}
\gamma^{t}=\frac{1}{\sqrt{f(r)}}\left(\begin{array}{cc}
i & 0 \\
0 & -i
\end{array}\right), & \gamma^{\theta}=\sqrt{g^{\theta \theta}}\left(\begin{array}{cc}
0 & \sigma^{1} \\
\sigma^{1} & 0
\end{array}\right), \\
\gamma^{r}=\sqrt{g(r)}\left(\begin{array}{cc}
0 & \sigma^{3} \\
\sigma^{3} & 0
\end{array}\right), & \gamma^{\phi}=\sqrt{g^{\phi \phi}}\left(\begin{array}{cc}
0 & \sigma^{2} \\
\sigma^{2} & 0
\end{array}\right),
\end{array}
$$

with $\sqrt{g^{\theta \theta}}=1 / r$ and $\sqrt{g^{\phi \phi}}=1 / r \sin \theta . \sigma^{i}$ s are the Pauli matrices with $i=1,2,3$.

Our task is to find the solutions of (13). First substitute the wave function (15) and the matrices (17) in the generalized Dirac equation (13) and cancel the exponential factor. Since we are working with WKB approximation, the contributions from $\partial A, \partial B$, and high orders of $\hbar$ are neglected. We finally obtain decoupled four Hamilton-Jacobi equations:

$$
\begin{aligned}
- & i A \frac{1}{\sqrt{f}} \partial_{t} I-B\left(1-\beta m^{2}\right) \sqrt{g} \partial_{r} I \\
- & A m \beta\left[g^{r r}\left(\partial_{r} I\right)^{2}+g^{\theta \theta}\left(\partial_{\theta} I\right)^{2}+g^{\phi \phi}\left(\partial_{\phi} I\right)^{2}\right] \\
+ & B \beta \sqrt{g} \partial_{r} I\left[g^{r r}\left(\partial_{r} I\right)^{2}+g^{\theta \theta}\left(\partial_{\theta} I\right)^{2}+g^{\phi \phi}\left(\partial_{\phi} I\right)^{2}\right] \\
+ & A m\left(1-\beta m^{2}\right)=0, \\
i B & \frac{1}{\sqrt{f}} \partial_{t} I-A\left(1-\beta m^{2}\right) \sqrt{g} \partial_{r} I \\
& -B m \beta\left[g^{r r}\left(\partial_{r} I\right)^{2}+g^{\theta \theta}\left(\partial_{\theta} I\right)^{2}+g^{\phi \phi}\left(\partial_{\phi} I\right)^{2}\right] \\
& +A \beta \sqrt{g} \partial_{r} I\left[g^{r r}\left(\partial_{r} I\right)^{2}+g^{\theta \theta}\left(\partial_{\theta} I\right)^{2}+g^{\phi \phi}\left(\partial_{\phi} I\right)^{2}\right] \\
& +B m\left(1-\beta m^{2}\right)=0, \\
& +i\left(1-\beta m^{2}\right) \sqrt{g^{\phi \phi}} \partial_{\phi} I+i \beta \sqrt{g^{\phi \phi}} \partial_{\phi} I \\
& \left.\left.+\left[\partial_{r} I\right)^{2}+g^{\theta \theta}\left(\partial_{\theta} I\right)^{2}+g^{\phi \phi}\left(\partial_{\phi} I\right)^{2}\right]\right\} \\
& \left.\left.\left.+i\left(1-\beta m^{2}\right) \sqrt{g^{\phi \phi}} \partial_{\phi} I+i \beta \sqrt{g^{\phi \phi}} \partial_{\phi} I\right)^{2}+g^{\theta \theta}\left(\partial_{\theta} I\right)^{2}+g^{\phi \phi}\left(\partial_{\phi} I\right)^{2}\right]\right\}=0, \\
& +\left(1-\beta m^{2}\right) \sqrt{g^{\theta \theta}} \partial_{\theta} I \\
& +\beta \sqrt{g^{\theta \theta}} \partial_{\theta} I\left[g^{r r}\left(\partial_{r} I\right)^{2}+g^{\theta \theta}\left(\partial_{\theta} I\right)^{2}+g^{\phi \phi}\left(\partial_{\phi} I\right)^{2}\right]
\end{aligned}
$$

To find the relevant solution, since the metric has a timelike killing vector, we perform the separation of variables as follows:

$$
I=-\omega t+W(r)+\Theta(\theta, \phi)
$$

where $\omega$ turns out to be the energy of the emitted particle. We insert (22) into (18)-(21) and first focus on the last two equations. They are identical after dividing, respectively, by $A$ and $B$ and can be rewritten as follows:

$$
\begin{aligned}
& \left(\sqrt{g^{\theta \theta}} \partial_{\theta} \Theta+i \sqrt{g^{\phi \phi}} \partial_{\phi} \Theta\right) \\
& \times\left[\beta g^{r r}\left(\partial_{r} W\right)^{2}+\beta g^{\theta \theta}\left(\partial_{\theta} \Theta\right)^{2}\right. \\
& \left.\quad+\beta g^{\phi \phi}\left(\partial_{\phi} \Theta\right)^{2}-\left(1-\beta m^{2}\right)\right]=0 .
\end{aligned}
$$


In the equation above, the value in the square bracket cannot vanish since $\beta$ is a small quantity representing the effects from quantum gravity. Therefore, the expression in the round brackets is zero and yields the solution of $\Theta$. In the previous work, though $\Theta$ has a complex solution (other than the trivial one $\Theta=$ constant) and gives rise to a contribution to the imaginary part of the action, it has no contribution to the tunnelling rate. Therefore, (23) is simplified as

$$
\sqrt{g^{\theta \theta}} \partial_{\theta} \Theta+i \sqrt{g^{\phi \phi}} \partial_{\phi} \Theta=0 .
$$

After cancelling $A$ and $B,(18)$ and (19) are identical and give rise to

$$
A_{6}\left(\partial_{r} W\right)^{6}+A_{4}\left(\partial_{r} W\right)^{4}+A_{2}\left(\partial_{r} W\right)^{2}+A_{0}=0
$$

with

$$
\begin{aligned}
A_{6}= & \beta^{2} g^{3} f \\
A_{4}= & \beta g^{2} f\left(m^{2} \beta+2 \beta Q-2\right), \\
A_{2}=g f & {\left[\left(1-\beta m^{2}\right)^{2}\right.} \\
& \left.\quad+\beta\left(2 m^{2}-2 m^{4} \beta-2 Q+\beta Q^{2}\right)\right], \\
A_{0}= & -m^{2}\left(1-\beta m^{2}-\beta Q\right)^{2} f-\omega^{2}, \\
Q= & g^{\theta \theta}\left(\partial_{\theta} \Theta\right)^{2}+g^{\phi \phi}\left(\partial_{\phi} \Theta\right)^{2} .
\end{aligned}
$$

Using (24), we find $Q=0$. Neglecting the higher orders of $\beta$ and solving the above equations at the event horizon yields (In [36-38], the authors argued that the invariance under canonical coordinate transformation requires that the integral to calculate the imaginary part of $W(r)$ should be a loop rather than an open one-way integral. However, in our calculation, only the difference between the imaginary parts matters.)

$$
\begin{aligned}
W(r)= \pm & \frac{1}{\sqrt{g f}} \sqrt{m^{2}\left(1-2 \beta m^{2}\right) f+\omega^{2}} \\
& \times\left(1+\beta\left(m^{2}+\frac{\omega^{2}}{f}\right)\right) d r \\
= & \pm i \pi 2 M \omega\left(1+\frac{1}{2} \beta\left(3 m^{2}+4 \omega^{2}\right)\right)+(\text { real part }) .
\end{aligned}
$$

In the above equation, $f=g=1-(2 M / r)$. The real part is irrelevant to the tunnelling rate. The $+/-$ sign corresponds to outgoing/ingoing wave. Then, the tunnelling rate [39] of the spin-1/2 fermion crossing the horizon is

$$
\begin{aligned}
\Gamma & =\frac{P_{(\text {emission })}}{P_{(\text {absorption })}}=\frac{\exp \left(-2 \operatorname{Im} I_{+}\right)}{\exp \left(-2 \operatorname{Im} I_{-}\right)} \\
& =\frac{\exp \left(-2 \operatorname{Im} W_{+}-2 \operatorname{Im} \Theta\right)}{\exp \left(-2 \operatorname{Im} W_{-}-2 \operatorname{Im} \Theta\right)} \\
& =\exp \left[-8 \pi M \omega\left(1+\frac{1}{2} \beta\left(3 m^{2}+4 \omega^{2}\right)\right)\right] .
\end{aligned}
$$

This is the Boltzmann factor with Hawking temperature:

$$
\begin{aligned}
T & =\frac{1}{8 \pi M\left(1+(1 / 2) \beta\left(3 m^{2}+4 \omega^{2}\right)\right)} \\
& =\left[1-\frac{1}{2} \beta\left(3 m^{2}+4 \omega^{2}\right)\right] T_{0},
\end{aligned}
$$

where $T_{0}=1 / 8 \pi M$ is the original Hawking temperature. It shows that there is a small correction to the Hawking temperature and the correction value is dependent not only on the black hole's mass but also on the mass and energy of emitted fermions. This property has been obtained in the literature. In [3], energy conservation is enforced by dynamical geometry and the tunnelling rate is found to be $\Gamma=\exp [-8 \pi \omega(M-$ $\omega / 2)]$. Then, the corrected Hawking temperature is $T=$ $1 /(8 \pi M-4 \pi \omega)$, where the leading correction to the Hawking temperature is related to the energy of emitted particles. To address the effects of quantum gravity, the authors of $[30,40]$ adopted the modified commutation relation between the radial coordinate and the conjugate momentum. They studied the quantum tunnelling of scalar particles in the Schwarzschild black hole. The tunnelling rate was derived as $\Gamma=\exp \left[-8 \pi M \omega+4 \pi \omega^{2}\left(3 M a l_{p}+1\right)-8 \pi \omega^{3}\left((7 / 3) M a^{2} l_{p}^{2}+\right.\right.$ $\left.\left.(4 / 3) a l_{p}\right)+20 \pi a^{2} l_{p}^{2} \omega^{4}+0\left(a^{2} l_{p}^{4}\right)\right]$. Thus, the correction to the Hawking temperature is also related to the black hole's mass and the particle's energy.

It is of interest to note that in (29) the quantum correction slows down the increase of the temperature during the radiation. This correction therefore causes the radiation cease at some particular temperature, leaving the remnant mass. To estimate the residue mass, it is enough to consider massless particles. The temperature stops increasing when

$$
(M-d M)\left(1+\beta \omega^{2}\right) \simeq M .
$$

Then with the observation $d M=\omega$ and $\beta=\beta_{0} / M_{p}^{2}$, where $M_{p}$ is the Planck mass and $\beta_{0}<10^{34}[41,42]$ is a dimensionless parameter marking quantum gravity effects, we can get

$$
M_{\mathrm{Res}} \simeq \frac{M_{p}^{2}}{\beta_{0} \omega} \gtrsim \frac{M_{p}}{\beta_{0}}, \quad T_{\mathrm{Res}} \lesssim \frac{\beta_{0}}{8 \pi M_{p}},
$$

where we have assumed that the maximal energy of the radiated particle is $\omega \simeq M_{p}$. This result is consistent with those obtained in [29, 43-45]. Compared with previous results, our calculation explicitly shows how the residue mass of black holes arises due to quantum gravity effects. The singularity of black hole evaporation is then prevented by the quantum gravity correction.

\section{Discussion and Conclusion}

In this work, we modified the Dirac equation in curved spacetime to include the quantum gravity influence. To fulfill this purpose, we employed the generalized uncertainty principle model. This model is derived from the existence of minimal length which arises when combining quantum and gravity. 
We calculated the radiation of spin-1/2 particles in the 4dimensional Schwarzschild spacetime with Hamilton-Jacob method. The tunnelling rate and Hawking temperature were presented.

We found that the quantum gravity correction is related not only to the black hole's mass but also to the mass (energy) of emitted fermions. More interestingly, our result shows that the quantum gravity correction explicitly retards the temperature rising in the black hole evaporation. Therefore, at some point during the evaporation, the quantum correction balances the traditional temperature rising tendency. This leads to the existence of the remnants. We showed that the remnants is $M_{\text {Res }} \geqslant M_{p} / \beta_{0}$, where $M_{p}$ is the Planck mass and $\beta_{0}<10^{34}$ from simple electroweak consideration. Therefore, the classical thermodynamics singularity can be avoided and a residue temperature $T_{\text {Res }} \leqslant \beta_{0} / 8 \pi M_{p}$ of black holes exists.

We use the 4-dimensional Schwarzschild metric in this work. It is known that the WKB type of approximation is basically the same as working with a 1+1-dimensional spacetime [46-48]. As a consequence, all large nonextremal black holes look basically the same (like Rindler space). It is also true for the charged and rotating black holes. Therefore, the remnants can be also found in other geometries. It is of interest to employ these geometries in the studies. In our calculation, we keep only the leading order of $\hbar$ and $\beta=$ $\beta_{0} / M_{p}^{2}$. It is expected that higher orders of corrections may give more information in the future work.

\section{Acknowledgments}

Deyou Chen and Houwen Wu are very grateful for Professor S. Q. Wu and Prof. Professor Wang for their useful discussions. This work is supported in part by the NSFC (Grants nos. 11205125, 11175039, and 11375121), Sichuan Province Science Foundation for Youths (Grant no. 2012JQ0039), and China West Normal University Foundation (Grant no. 11B005).

\section{References}

[1] S. W. Hawking, "Particle creation by black holes," Communications in Mathematical Physics, vol. 43, p. 199, 1975.

[2] P. Kraus and F. Wilczek, "Self-interaction correction to black hole radiance," Nuclear Physics B, vol. 433, no. 2, pp. 403-420, 1995.

[3] M. K. Parikh and F. Wilczek, "Hawking radiation as tunneling," Physical Review Letters, vol. 85, p. 5042, 2000.

[4] J. Zhang and Z. Zhao, "Hawking radiation of charged particles via tunneling from the Reissner-Nordström black hole," Journal of High Energy Physics, no. 10, pp. 1377-1383, 2005.

[5] Q. Q. Jiang, S. Q. Wu, and X. Cai, "Hawking radiation as tunneling from the Kerr and Kerr-Newman black holes," Physical Review D, vol. 73, Article ID 064003, 2006.

[6] M. Angheben, M. Nadalini, L. Vanzo, and S. Zerbini, "Hawking radiation as tunneling for extremal and rotating black holes," Journal of High Energy Physics, no. 5, pp. 274-291, 2005.

[7] K. Srinivasan and T. Padmanabhan, "Particle production and complex path analysis," Physical Review D, vol. 60, Article ID 24007, 1999.
[8] B. Chatterjee, A. Ghosh, and P. Mitra, "Tunnelling from black holes and tunnelling into white holes," Physics Letters B, vol. 661, p. 307, 2008.

[9] E. C. Vagenas, "Semiclassical corrections to the BekensteinHawking entropy of the BTZ black hole via self-gravitation," Physics Letters B, vol. 533, no. 3-4, pp. 302-306, 2002.

[10] R. Kerner and R. B. Mann, "Fermions tunnelling from black holes," Classical and Quantum Gravity, vol. 25, Article ID 095014, 2008.

[11] R. Kerner and R. B. Mann, "Charged Fermions tunnelling from Kerr-Newman black holes," Physics Letters B, vol. 665, p. 277, 2008.

[12] R. Li and J. R. Ren, "Dirac particles tunneling from BTZ black hole," Physics Letters B, vol. 661, p. 370, 2008.

[13] D. Y. Chen, Q. Q. Jiang, and X. T. Zu, "Hawking radiation of Dirac particles via tunnelling from rotating black holes in de Sitter spaces," Physics Letters B, vol. 665, p. 106, 2008.

[14] Q. Q. Jiang, "Fermions tunnelling from GHS and non-extremal D1-D5 black holes," Physics Letters B, vol. 666, p. 517, 2008.

[15] R. Di Criscienzo and L. Vanzo, "Fermion tunneling from dynamical horizons," Europhysics Letters, vol. 82, Article ID 60001, 2008.

[16] K. Nozari and S. H. Mehdipour, "Hawking radiation as quantum tunneling from a noncommutative Schwarzschild black hole," Classical and Quantum Gravity, vol. 25, no. 17, Article ID 175015, 2008.

[17] P. K. Townsend, "Small-scale structure of spacetime as the origin of the gravitational constant," Physical Review D, vol. 15, no. 10, pp. 2795-2801, 1977.

[18] D. Amati, M. Ciafaloni, and G. Veneziano, "Can spacetime be probed below the string size?" Physics Letters B, vol. 216, no. 1-2, pp. 41-47, 1989.

[19] K. Konishi, G. Paffuti, and P. Provero, "Minimum physical length and the generalized uncertainty principle in string theory," Physics Letters B, vol. 234, no. 3, pp. 276-284, 1990.

[20] L. J. Garay, "Quantum gravity and minimum length," International Journal of Modern Physics A, vol. 10, p. 145, 1995.

[21] G. Amelino-Camelia, "Relativity in spacetimes with short-distance structure governed by an observer-independent (Planckian) length scale," International Journal of Modern Physics D, vol. 11, no. 1, pp. 35-59, 2002.

[22] A. Kempf, G. Mangano, and R. B. Mann, "Hilbert space representation of the minimal length uncertainty relation," Physical Review D, vol. 52, p. 1108, 1995.

[23] A. Kempf, "Nonpointlike particles in harmonic oscillators," Journal of Physics A, vol. 30, p. 2093, 1997.

[24] F. Brau, "Minimal length uncertainty relation and Hydrogen atom," Journal of Physics A, vol. 32, p. 7691, 1999.

[25] A. F. Ali, S. Das, and E. C. Vagenas, "Discreteness of space from the generalized uncertainty principle," Physics Letters B, vol. 678, p. 497, 2009.

[26] W. Kim, E. J. Son, and M. Yoon, "Thermodynamics of a black hole based on a generalized uncertainty principle," Journal of High Energy Physics, vol. 2008, p. 35, 2008.

[27] K. Nozari and S. H. Mehdipour, "Parikh-Wilczek tunneling from noncommutative higher dimensional black holes," Journal of High Energy Physics, vol. 2009, p. 61, 2009.

[28] L. Xiang and X. Q. Wen, "A heuristic analysis of black hole thermodynamics with generalized uncertainty principle," Journal of High Energy Physics, vol. 2009, p. 46, 2009. 
[29] R. Banerjee and S. Ghosh, "Generalised uncertainty principle, remnant mass and singularity problem in black hole thermodynamics," Physics Letters B, vol. 688, p. 224, 2010.

[30] K. Nozari and S. Saghafi, "Natural cutoffs and quantum tunneling from black hole horizon," Journal of High Energy Physics, vol. 2012, p. 5, 2012.

[31] A. F. Ali, "No existence of black holes at LHC due to minimal length in quantum gravity," Journal of High Energy Physics, vol. 2012, p. 67, 2012.

[32] B. Majumder, "Quantum black hole and the modified uncertainty principle," Physics Letters B, vol. 701, p. 384, 2011.

[33] S. Hossenfelder, M. Bleicher, S. Hofmann, J. Ruppert, S. Scherer, and H. Stöcker, "Signatures in the Planck regime," Physics Letters B, vol. 575, no. 1-2, pp. 85-99, 2003.

[34] K. Nozari and M. Karami, "Minimal length and generalized Dirac equation," Modern Physics Letters A, vol. 20, p. 3095, 2005.

[35] S. Hossenfelder, M. Bleicher, S. Hofmann, J. Ruppert, S. Scherer, and H. Stocker, "Signatures in the Planck regime," Physics Letters $B$, vol. 575 , p. 85, 2003.

[36] E. T. Akhmedov, V. Akhmedova, D. Singleton, and T. Pilling, "Thermal radiation of various gravitational backgrounds," International Journal of Modern Physics A, vol. 22, no. 8-9, pp. 17051715, 2007.

[37] B. D. Chowdhury, "Problems with tunneling of thin shells from black holes," Pramana, vol. 70, no. 1, pp. 3-26, 2008.

[38] E. T. Akhmedov, V. Akhmedova, and D. Singleton, "Hawking temperature in the tunneling picture," Physics Letters B, vol. 642, p. 124, 2006.

[39] P. Mitra, "Hawking temperature from tunnelling formalism," Physics Letters B, vol. 648, p. 240, 2007.

[40] K. Nozari and S. H. Mehdipour, "Quantum gravity and recovery of information in black hole evaporation," Europhysics Letters, vol. 84, p. 20008, 2008.

[41] P. Wang, H. Yang, and X. Zhang, "Quantum gravity effects on statistics and compact star configurations," Journal of High Energy Physics, vol. 2010, p. 43, 2010.

[42] P. Wang, H. Yang, and X. Zhang, "Quantum gravity effects on compact star cores," Physics Letters B, vol. 718, p. 265, 2012.

[43] R. J. Adler, P. Chen, and D. I. Santiago, "The generalized uncertainty principle and black hole remnants," General Relativity and Gravitation, vol. 33, p. 2101, 2001.

[44] P. Chen and R. J. Adler, "Black hole remnants and dark matter," Nuclear Physics B: Proceedings Supplements, vol. 124, pp. 103106, 2003.

[45] L. Xiang, "A note on the black hole remnant," Physics Letters B, vol. 647, p. 207, 2007.

[46] S. P. Robinson and F. Wilczek, "Relationship between Hawking radiation and gravitational anomalies," Physical Review Letters, vol. 95, no. 1, Article ID 011303, 2005.

[47] S. Iso, H. Umetsu, and F. Wilczek, "Hawking radiation from charged black holes via gauge and gravitational anomalies," Physical Review Letters, vol. 96, Article ID 151302, 2006.

[48] S. Iso, H. Umetsu, and F. Wilczek, "Anomalies, Hawking radiations, and regularity in rotating black holes," Physical Review D, vol. 74, Article ID 044017, 2006. 

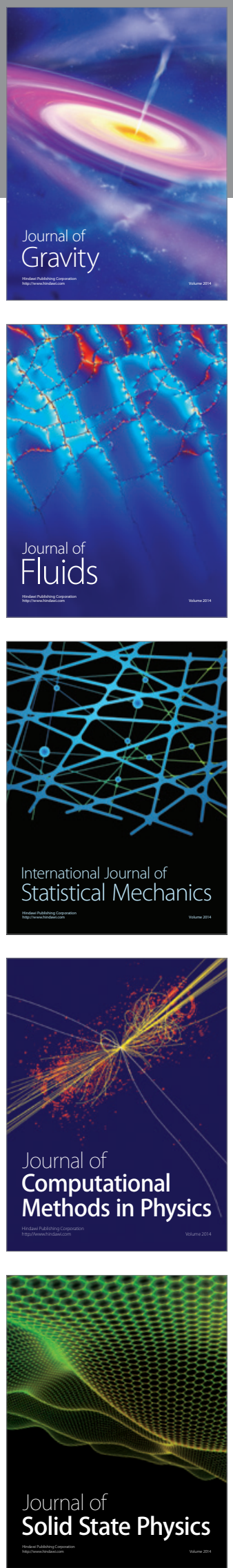

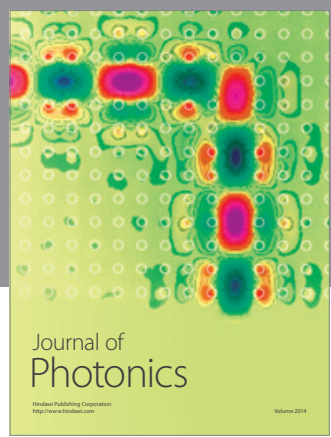

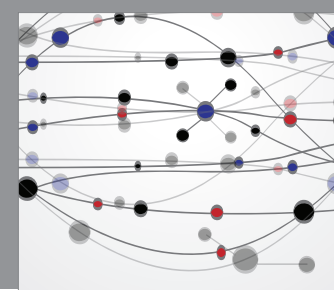

The Scientific World Journal

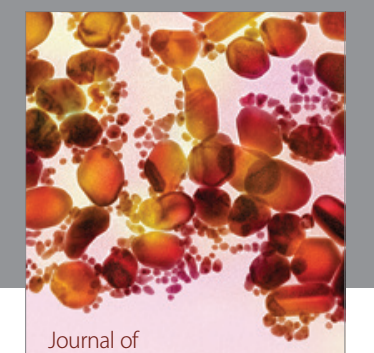

Soft Matter
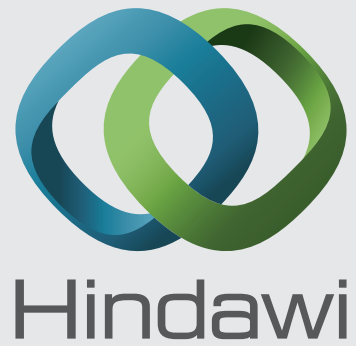

Submit your manuscripts at

http://www.hindawi.com
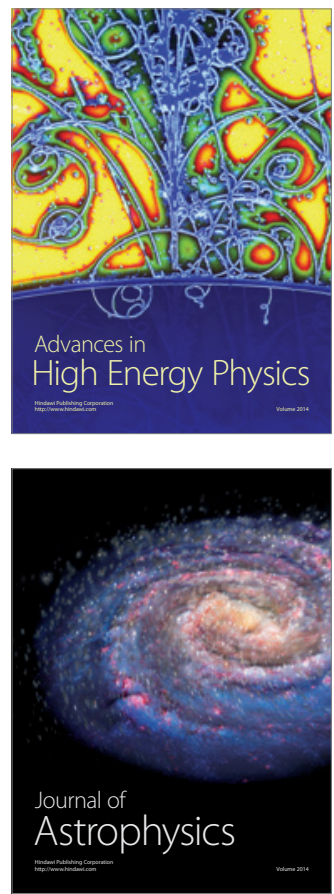
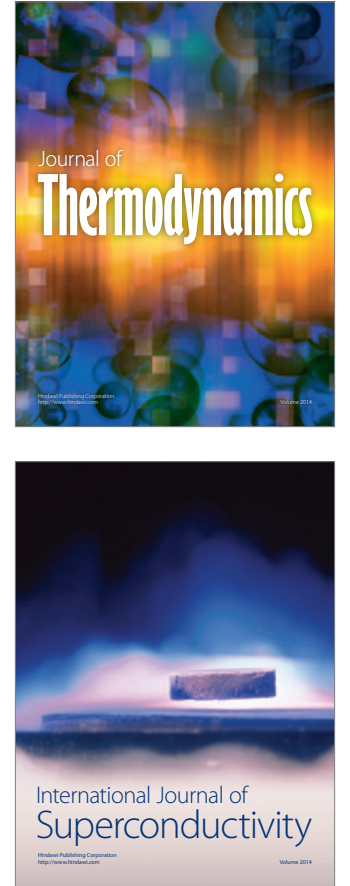
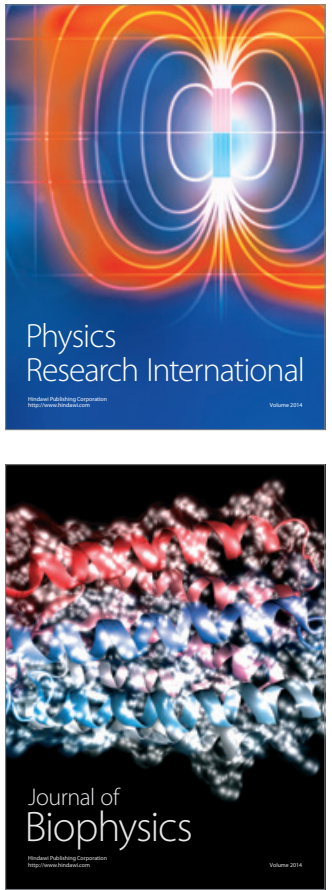
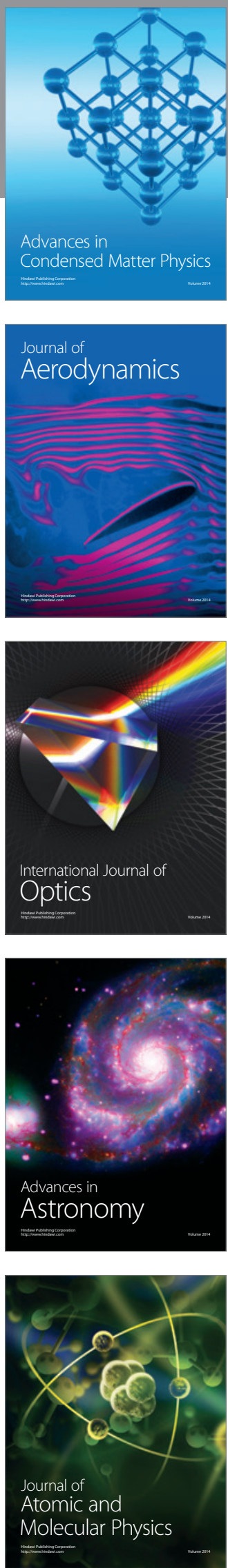